\title{
Use clinical pulmonary infection tests for definition of ventilator associated pneumonia in comatose ill neurosurgical patients
}

\author{
Sagimbayev A., Mustafin A., Mustafin A., Medical University Astana, \\ Dept of Anaesthesiology \& Intensive Care, Astana, Kazakhstan
}

\section{Background:}

Ventilator associated pneumonia (VAP) remains the most common nosocomial infection in the ICU with a very high morbidity, mortality and cost of treatment. Clinical pulmonary infection tests (CPIT) can be used prospectively to diagnosis VAP, so as to initiate early treatment and prevent mortality. Most studies indicate that the CPIT has limited value to diagnose VAP. We conducted a prospective study to detect VAP using CPIT in neurosurgical patients.

\section{Materials and Method:}

After approval of Ethics Committee, 76 consecutive neurosurgical patients who required ventilatory support for more than 48 hours were studied. CPIT was calculated every day and when the CPIT was $\geq 6$, mini-BAL was taken by catheter in catheter technique and was analyzed for microorganism.

\section{Results:}

A total of 29 VAP episodes were identified using CPIT $(\geq 6)$ during the study period, of which only 13 patient's tracheal aspirate were positive for microorganism. The incidence rate expressed as the total number of VAP episodes per 1000 ventilation days using CPIT and tracheal aspirate culture was 39.7(29/889) and 23.87(19/889) respectively in this cohort, but overall our ICU VAP rate in neurosurgical patients using CPIT and tracheal aspirate culture was 15.19(29/1909) and 9.42(18/1909) respectively. Four patients were found to have early VAP ( $\leq 5$ days of MV) and rest had late VAP.The most common organism was Acinetobacter Baumanni, followed by Enterobacteracae. Early VAP was caused by Enterobacteracae and Acinetobactor causing late VAP.

\section{Conclusion:}

CPIT can be a fairly good method to diagnose VAP in comatose ill neurosurgical patients, when used reasonably and at the same time can help to restrict unnecessary antibiotic use. 